\title{
Pilot Log Anonymization in GlideinMonitor
}

\section{Mirica Yancey, Valparaiso University - SIST Intern}

FERMILAB-POSTER-20-114-SCD

\section{Introduction}

GlideinWMS is a pilot-based resource provisioning tool for distributed High

Throughput Computing

- Provides reliable and uniform virtual clusters to run scientific computations (analysis, simulations, reconstructions etc.)

- Provisions computers from many sources (local clusters, Grid, Cloud, Supercomputers)

\section{GlideinMonitor}

- Web application to view GlideinWMS's log files

- User interface tool

- Useful for searches and decoding log content

- Contains an efficient, managed archive of log files

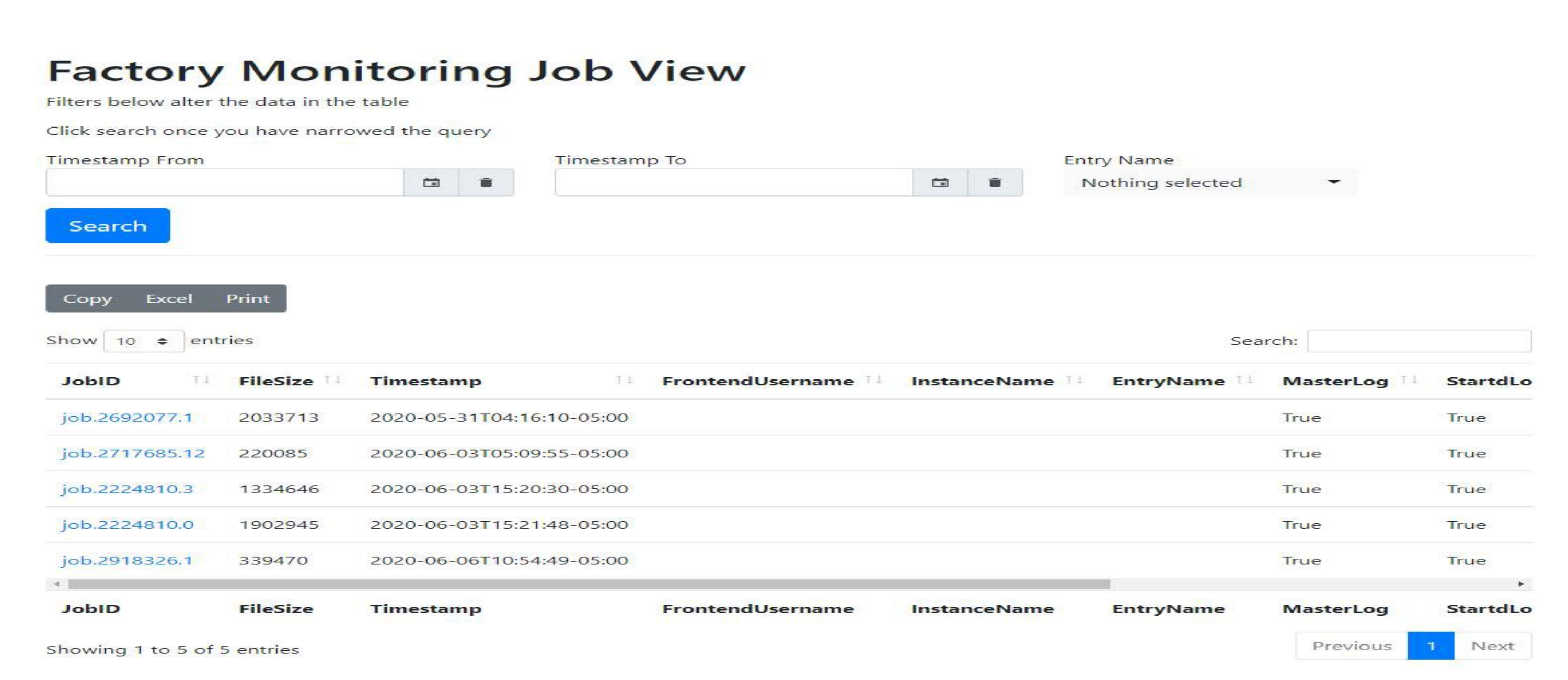

Figure 1 - GlideinMonitor Homescreen

\section{Problem}

Log files contain sensitive information that makes the logs less secure and decreases the ease of distribution amongst developers.

\section{Objective}

Create a script that:

- Locates the job submitter's IP Address - Locates any identifiers of the job submitter - Filters those values with non-identifiable information

In order to:

-Protect User Information

-Allow easier distribution to developers

\section{Methods}

Identified what the personal information was and then cross-referenced files to ensure there was a consistent reference for this information in order to search and retrieve it.

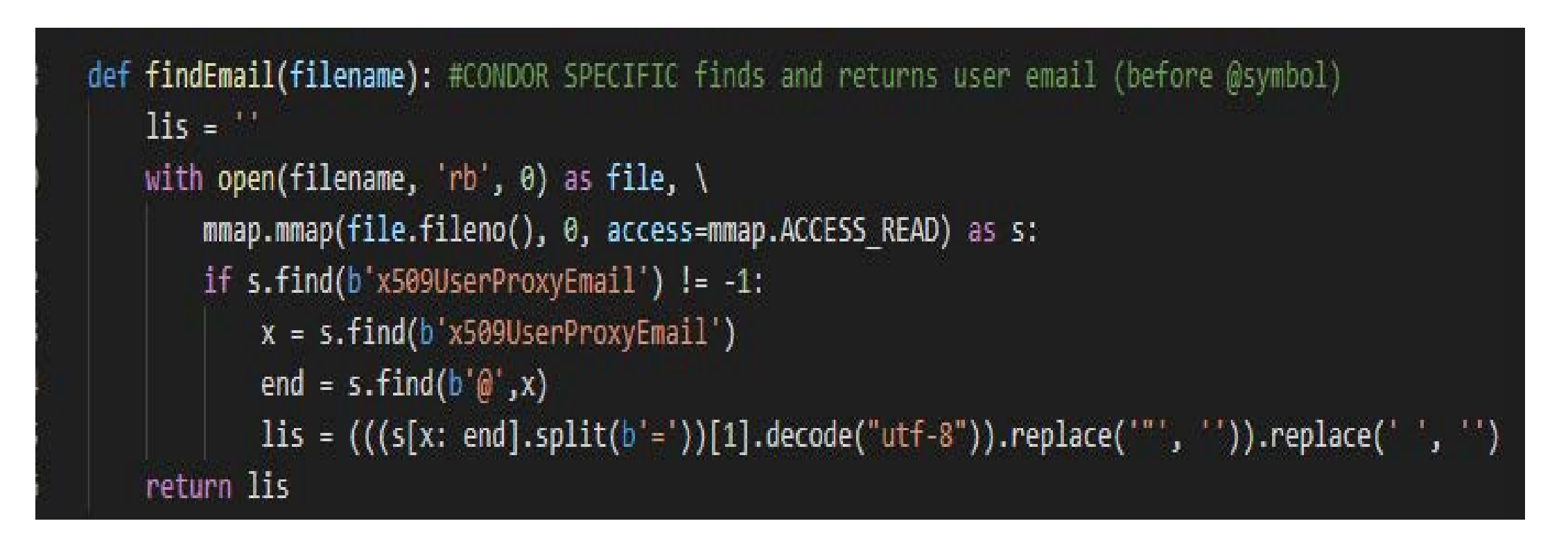

Figure 2 - Email Identifier Script

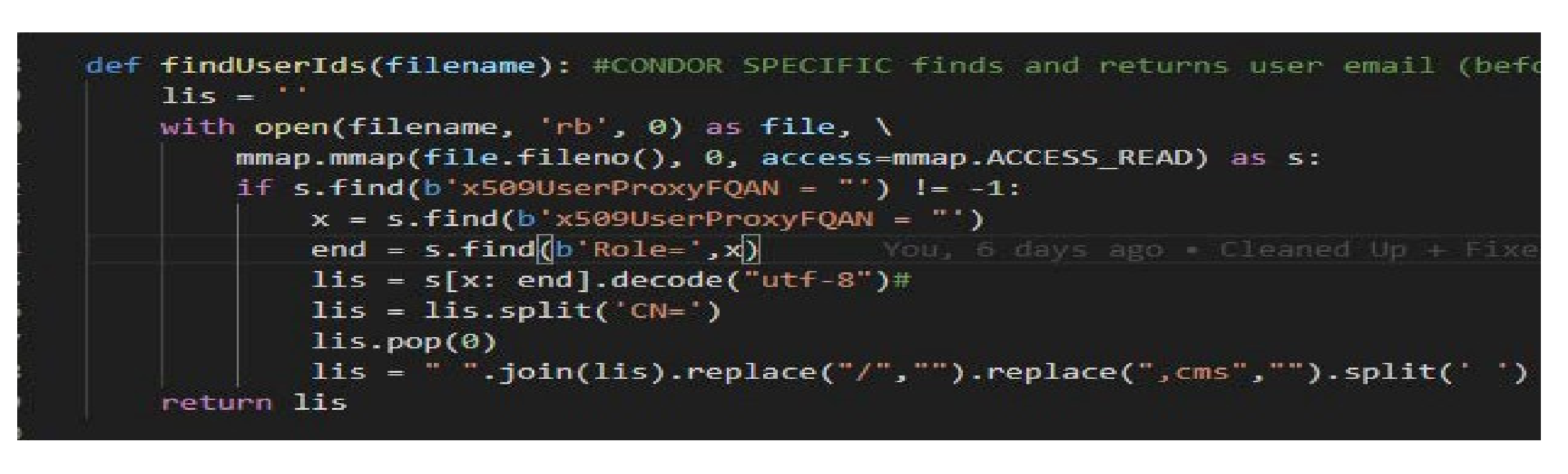

Figure 3 - User Information Identifier Script Created a method to replace this information and followed software engineering standards: added docstrings and unit tests to insure clarity and quality

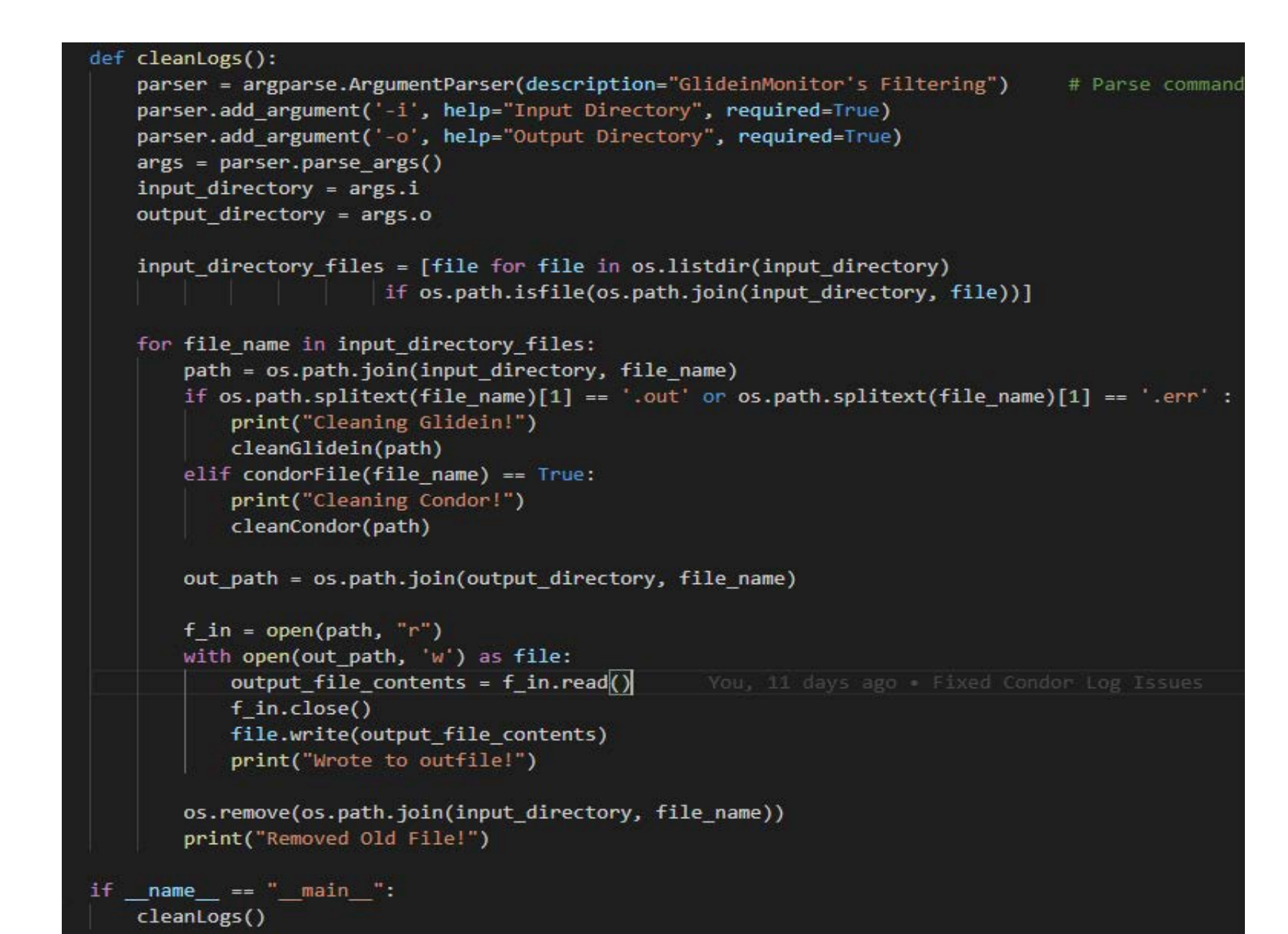

Figure 4-Main Filtering Method

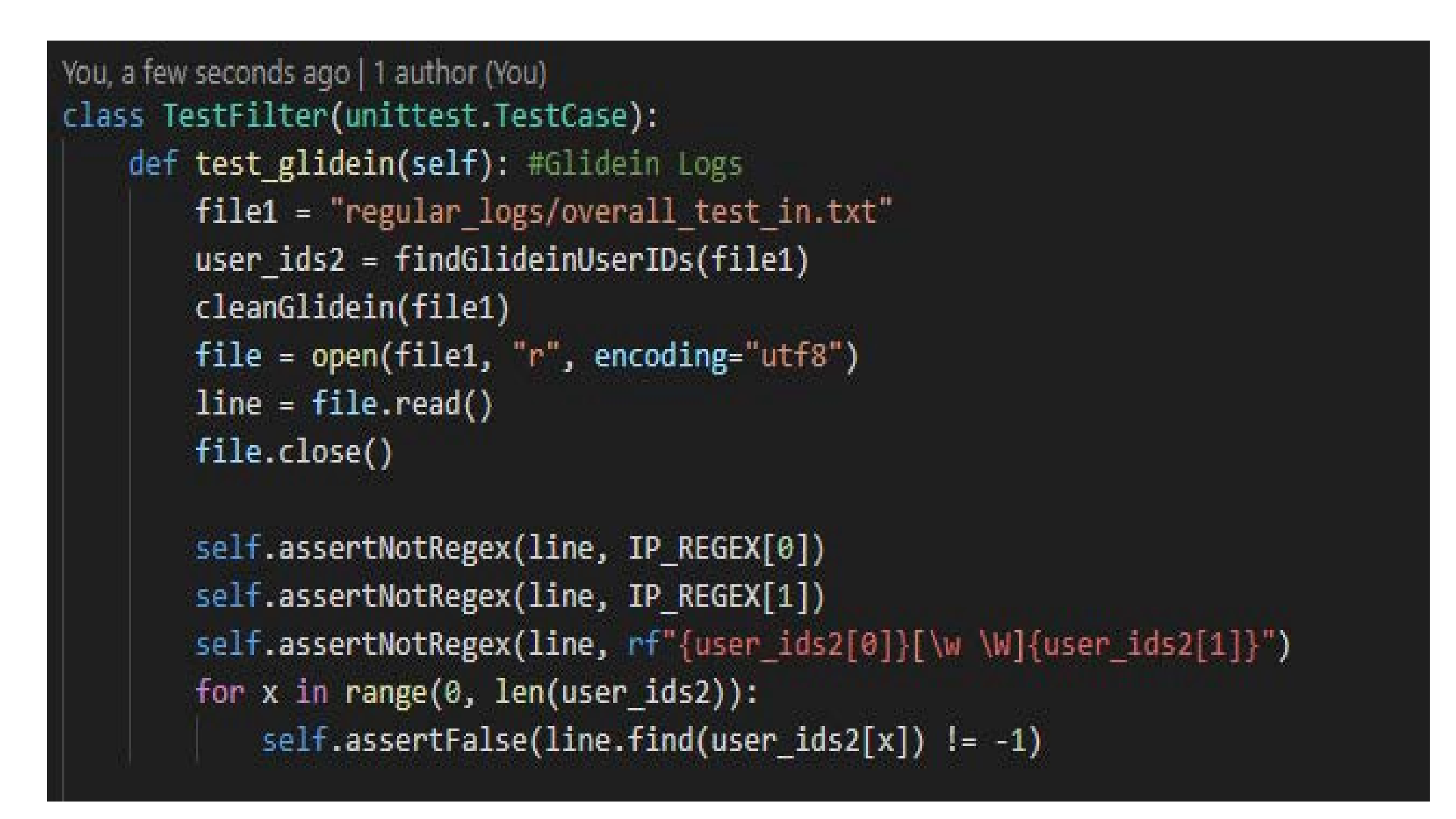

Figure 5 - Glidein Unit Test

\section{Results}

- Filtered logs contain no personal identifiers

- Script tested individually

- Script integrated into GlideinMonitor and included in RPM release

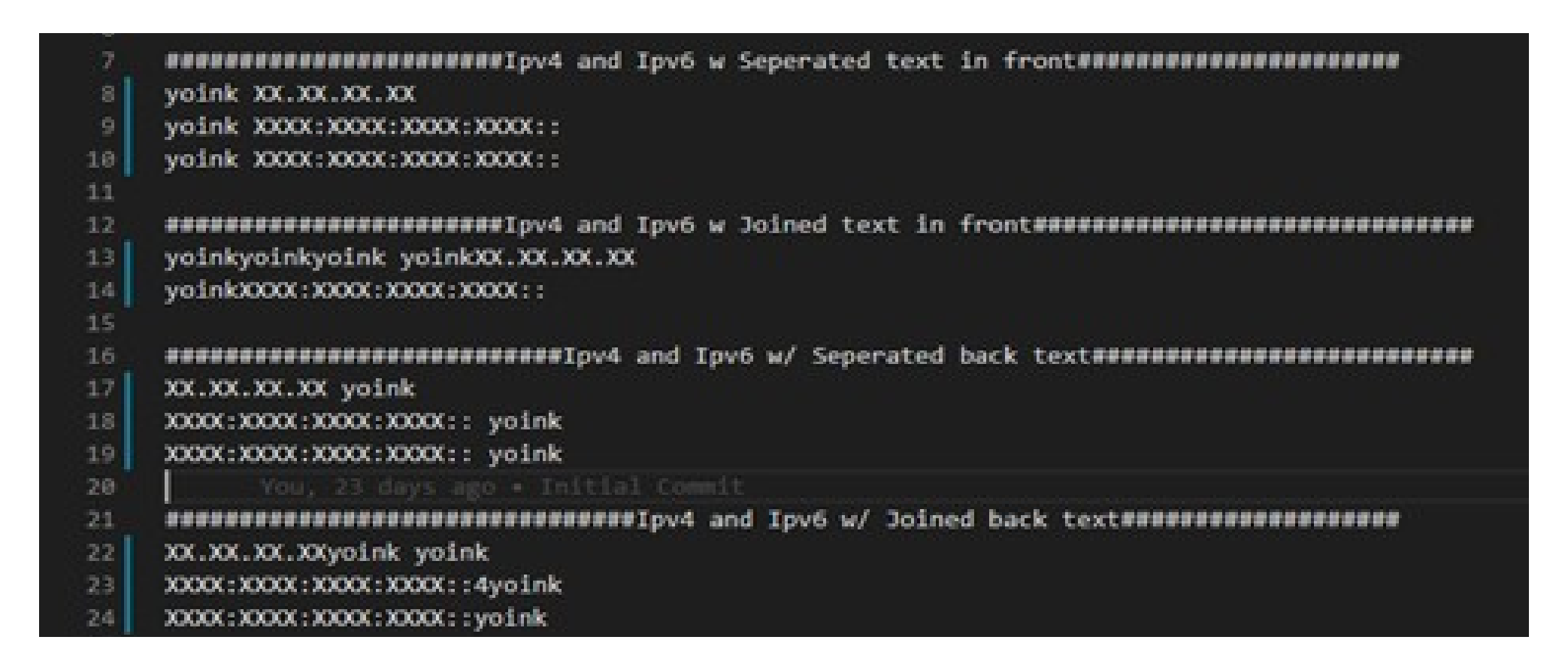

Figure 6 - Filtered Test File

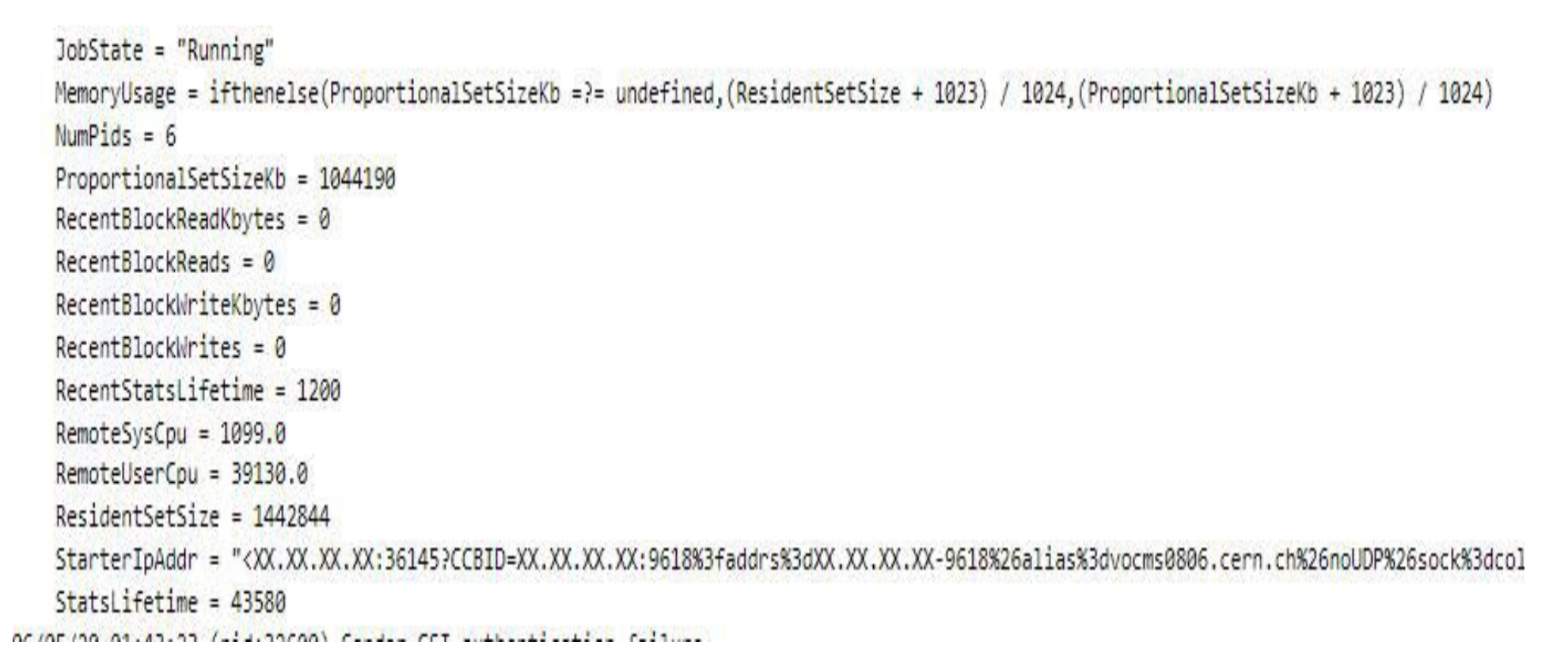

Figure 7 - Filtered IP Address in Log File

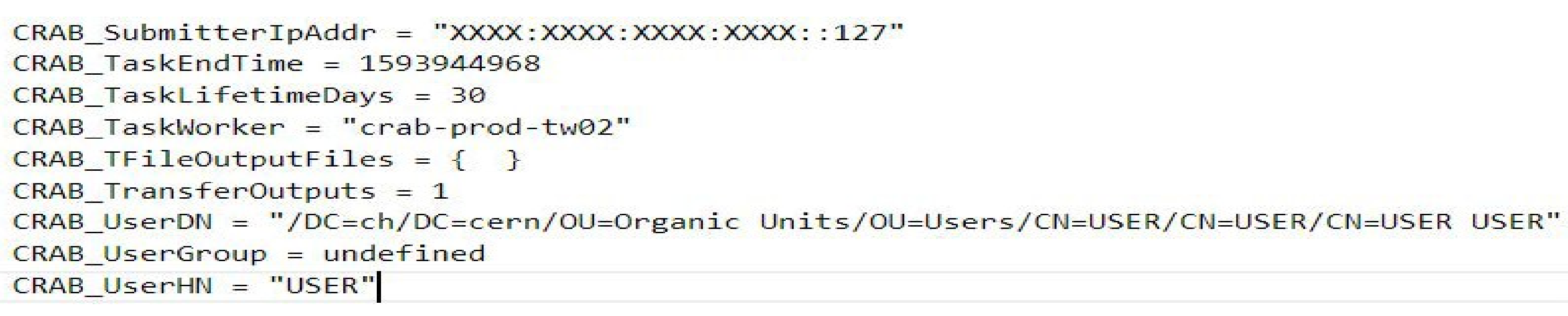

Figure 8 - Filtered User Identifiers in Log File

\section{Acknowledgements}

Thank you to my supervisor Marco Mambelli , the GlideinWMS team, and my SIST Mentoring team for consistently making me feel welcome and heard. Thank you for the belief in my skill set and the time you dedicated towards helping me grow that set. In addition to that, thank you Fermilab for the amazing opportunity you provided to me and the other interns this summer. This manuscript has been authored by Fermi Research Alliance, LLC under Contract No. DE-AC02-07CH11359 with the U.S. Department of Energy, Office of Science, Office of High Energy Physics 